\title{
APRENDizaJe, CUlTURA Y DESARROLLO. UNA APROXIMACIÓN INTERDISCIPLINARIA: RESEÑA
}

\author{
Ronal Iván Yepes Serrano \\ Estudiante de Estudios Literarios, \\ Pontificia Universidad Javeriana, \\ Bogotá, Colombia \\ yepes.ronal@javeriana.edu.co
}

Título: Aprendizaje, cultura y desarrollo. Una aproximación interdisciplinaria. Editoras: Susana Frisancho, Maria Teresa Moreno, Patricia Ruiz-Bravo y Virginia Zavala

Año de publicación: 2017, 2. ${ }^{a}$ edición. Casa editorial: Fondo Editorial Pontificia Universidad Católica del Perú ISBN: 9786123170097

No. de páginas: 240

Formato: Epub
Esta recopilación de trabajos de investigadores latinoamericanos muestra diferentes perspectivas de algunos estudios de literacidad en educación que se han hecho en algunas partes de América. Las editoras recopilan y ponen en diálogo estudios de caso que van en distintos órdenes de los saberes. Aprender a leer y escribir significa, entre otros, interactuar con otros lectores y escritores y ver el conocimiento forjado en el uso cotidiano de la lengua escrita como un recurso importante (Street, 2005). Requiere, a su vez, contar con la participación de usuarios, el conocimiento de los discursos por parte de estos, el aprendizaje de modos de leer e interpretar y de vincularse en eventos comunicativos (Gumperz \& Hymes, 1972).

Estas investigaciones abordan modos de aproximación a prácticas de lenguaje situadas, además de mostrar la íntima relación entre el desarrollo humano y la cultura, a partir de una revisión de lo que implica aprender en contextos diversos. En esta obra se analizan prácticas deaprendizajeen contextos naturales situados que muestran la importancia de reconocer los procesos internos de los aprendices, sus capacidades lingüísticas y cognitivas, sus creencias, su ambiente, así como no restringir las posibilidades de investigación a la escuela, sino extenderlas a las esferas de la vida regular (la casa, el trabajo, el juego, las tareas domésticas en la familia y en la comunidad).

Con estos estudios se evidencia que los modos como la gente interactúa con el lenguaje están relacionados con patrones culturales que reflejan formas de vida y visiones de mundo. De ahí la vinculación de estas investigaciones con los estudios de literacidad, dado que se plantea el carácter heterogéneo de los usos orales y escritos de la lengua y se asumen en el marco de relaciones sociales. En las diferentes prácticas de apropiación del conocimiento analizadas en la obra se da valor a las relaciones que se establecen con los otros (lectores y escritores enmarcados en sus comunidades), así como a los modos de representación y los instrumentos mediadores (simbólicos y humanos) que desde una mirada integral posibilitan el estudio de las diversas prácticas de literacidad instaladas en la cultura. 
Las editoras de la recopilación constituyen un equipo multidisciplinario; profesoras e investigadoras de la Pontificia Universidad Católica de Perú (PUCP), Susana Frisancho, hace parte del departamento de psicología; Virginia Zavala, del departamento de lingüística; y Patricia Ruiz Bravo López, del departamento de ciencias sociales, mientras que María Teresa Moreno es investigadora independiente, en temas educativos relacionados con educación inclusiva, desarrollo curricular, capacitación docente y evaluación. Esa unión propicia una mirada interdisciplinaria y dialógica, con aportes desde los campos de la psicología, la lingüística, la pedagogía y las ciencias sociales.

El libro Aprendizaje, cultura y desarrollo: una aproximación interdisciplinaria, publicado por el Fondo Editorial de la Pontificia Universidad Católica del Perú en 2011 y reimpreso en 2017, incluye estudios relacionados con los procesos de aprendizaje desde los Nuevos Estudios de Literacidad (NEL, en adelante), es decir, que se aportan investigaciones empíricas y conceptuales sobre prácticas de lectura y escritura situadas, que varían de acuerdo a los contextos en que se actualizan y se relacionan con estructuras de poder (Zavala et al., 2004). A través de los capítulos que componen esta obra se hace énfasis en las relaciones de la comunidad con el contexto histórico, político y geográfico y las formas particulares de leer y escribir que hay en los diferentes espacios en los que los sujetos desarrollan su actividad educativa, vinculada a procesos y estilos de aprendizaje en clave de prácticas sociales. Se abordan las complejas relaciones entre los procesos de aprendizaje, las prácticas letradas y su vínculo con prácticas culturales y procesos de socialización de niños y niñas. Hay que anotar que las comunidades a las que se refieren los estudios se desenvuelven en distintos lugares, contextos del territorio americano (Estados Unidos, México, Perú y Argentina). Además, las comunidades hacen parte de unos campos más amplios en los que se investigan prácticas de literacidad con relación a la interculturalidad que pueda haber entre países, regiones y estratos socioeconómicos (dentro y fuera de cada país). El libro está dividido en cinco secciones: marco conceptual general sobre aprendizaje, cultura y desarrollo; Socialización temprana y desarrollo infantil; desencuentros e inequidades en la transición hogar escuela; desafíos en la educación intercultural bilingüe y prácticas letradas y multimodales más allá de la escuela.

La primera parte aborda el tema de la caracterización histórico-cultural para una educación con intenciones de ver las diferentes prácticas y formas de educar y aprender en grupos étnicos en Estados Unidos y México. Esto se evidencia en los trabajos de Bárbara Rogoff y Kris Gutiérrez, "Maneras culturales de aprender: rasgos individuales o repertorios de práctica”, y en el trabajo de Ruth Paradise, “Cómo educan los indígenas a sus hijos? El cómo y el porqué del aprendizaje en la familia y en la comunidad".

El capítulo de Rogoff y Gutiérrez (2017) está orientado hacia las regularidades en los modos de aprender de individuos que son miembros de grupos étnicos, a quienes poco han tenido en cuenta en las escuelas de los Estados Unidos. Estos grupos son estudiantes afroamericanos, latinos e indígenas. Con una perspectiva histórico-cultural, el trabajo menciona cómo las investigaciones sobre estilos culturales de aprendizaje aparecieron en Estados Unidos en la década de 1960 con el ánimo de disminuir la inequidad y las escasas experiencias escolares de los estudiantes. Con este enfoque se busca dejar atrás el "modelo del déficit", el cual está principalmente enfocado a nivelar las supuestas deficiencias de los estudiantes que por su lengua o su procedencia geográfica se consideraban en un nivel escolar inferior con respecto a los que tenían, por ejemplo, como lengua original el inglés y pertenecían a familias de ciudad o con mayores ingresos. Por otro lado, Ruth Paradise plantea una perspectiva de la educación en espacios no convencionales, no oficiales, es decir, en lugares en los que no hay una normativa institucional establecida como la escuela propiamente dicha, sino, por ejemplo, aquella que se da en contextos familiares y comunitarios. La autora muestra cómo esas prácticas si bien no son escolarizadas en los grupos indígenas estudiados no son un 
"fenómeno espontáneo", sino que pueden ser sistemáticas y son prácticas educativas ancestrales que la gente desarrolla para tener un lugar como sujeto en las actividades de la comunidad.

En la sección "Socialización temprana y desarrollo infantil" se encuentran los trabajos: "Relaciones de afecto orientadas al bienestar colectivo en la socialización temprana”, de Fernando García, y “'Calibrando’ la atención: directivos, adiestramiento y responsabilidad en el trabajo doméstico de los niños mayas zinacantecos", de Lourdes de León. El primero se centra en la descripción de prácticas de cuidado de los niños de la comunidad de Aucará (zona sur andina de Perú) en el marco de las relaciones de afecto en la vida familiar y comunitaria. En esta descripción se integran explicaciones de la variante quechua hablada en la comunidad. En el otro capítulo se analizan las prácticas comunicativas que dirigen y estructuran la atención de los niños en el trabajo doméstico en el contexto de familias zinacantecas (Chiapas, México). Especialmente, el estudio se enfoca en formas discursivas directivas y estrategias interactivas implicadas en la negociación entre niños y adultos, las cuales resultan fundamentales en la organización de la atención y el aprendizaje.

La tercera sección "Desencuentros e inequidades en la transición hogar escuela" se centra en la transición que hay del entorno cotidiano de los educandos hacia el entorno oficial, institucionalizado, y se analiza el proceso de aprendizaje de la lectura y la escritura. Se incluye el trabajo de Patricia Ames "Empezando la escuela: las transiciones de los niños pequeños en diversos contextos socioculturales", se observa este proceso en niños peruanos indígenas y de otras etnias en zonas rurales y urbanas entre 5 y 6 años, en contextos culturales diferentes, puesto que ello permite acceder a una mirada comparativa y repensar la relación entre cultura y aprendizaje en el marco de las transiciones educativas que experimentan los niños. Concentrándose en el inicio de la escolaridad, Ames comprueba que los niños participan en una gran variedad de actividades cotidianas, que incluyen las actividades domésticas y labores agrícolas, en los casos rurales, mientras que los niños de las zonas urbanas realizan actividades de juego, separados de las labores domésticas. En esa misma línea se encuentra el trabajo de Rebeca Mejía-Arauz, Leslie Reese, Antonio Ray Bazán, Claude Goldenberg y Victoria Torres Armenta "El ambiente alfabetizador en familias y comunidades y su relación con el aprendizaje de la lecto-escritura”, en el cual se aborda la etapa de transición del entorno familiar a la escuela en los primeros grados escolares. Los investigadores plantean que el aprendizaje de la lectura y la escritura, si bien se desarrolla con ciertos valores específicos en el contexto particular de la escuela, también se da en la cotidianidad. Hay una conexión entre la vivencia y el aprendizaje que complejizan el acto mismo de aprender a leer y escribir: una configuración de valores, perspectivas y costumbres específicos en cada individuo o grupo de participantes. El estudio pone el acento en la relación entre escuela, familia y comunidad las cuales se configuran e intervienen en el desarrollo del lenguaje y las competencias lectoras y escritoras del niño.

En la sección Desafíos en la educación intercultural bilingüe, el foco está puesto sobre la educación bilingüe en las culturas indígenas latinoamericanas, en la traducción de contenidos académicos a las lenguas vernáculas. Desde esta investigación se repiensa la educación indígena desde una perspectiva de descolonización cultural e ideológica y se plantea la necesidad de una educación distinta que incluyera los conocimientos y valores de los pueblos indígenas. Los programas de educación intercultural bilingüe (EIB, en adelante), que se analizan en los trabajos incluidos en esta sección se desarrollaron principalmente en el Perú y abordan la diversificación de los currículos básicos desde enfoques como el de EIB, los NEL, la etnografía y la teoría de la actividad situada.

En el primer trabajo "¿Diversificar o interculturalizar el currículo?” de Lucy Trapnell, se destaca que los programas EIB que desarrollan organizaciones y ONG deben prestarle mayor atención a la relación conocimiento-poder si quieren contribuir al objetivo social y político de transformar 
las escuelas en espacios de empoderamiento de pueblos con una historia de marginación. La investigación deja en evidencia la necesidad de crear currículos educativos alternativos que consideren nuevas formas de abordar la realidad e introduzcan categorías de análisis e interpretación distintas a las propuestas por otras disciplinas. De otro lado, en el trabajo de Virginia Zavala "Deconstruyendo la educación intercultural bilingüe: los aportes de la sociolingüística crítica" se propone un "vuelco postestructuralista" que cambia la noción tradicional de la escuela, es decir, un lugar en el cual se produce y transmite la verdad. En este caso, la mirada está puesta sobre todo en el lenguaje desde el análisis crítico del discurso; así mismo, se reflexiona críticamente sobre el EIB con base en la sociolingüística crítica, en la medida en que se reconocen las relaciones de poder que subyacen a las formas en que el lenguaje representa y construye la realidad.

Por último, la sección Prácticas letradas más allá de la escuela, incluye los estudios "Literacidad híbrida en dos comunidades de Texas”, de María Teresa de la Piedra, y "Multimodalidad y apropiación de conocimientos en procesos de literacidad de adultos de baja escolaridad", de María del Carmen Lorenzatti. De la Piedra cuestiona las visiones homogeneizantes y deficitarias que existen sobre los inmigrantes mexicanos en los Estados Unidos. Plantea que la literacidad, en algunas reformas educativas en este contexto, es definida como un conjunto de prácticas y habilidades que los estudiantes de sectores sociales "minorizados" deben adquirir para aprobar los exámenes estandarizados obligatorios del estado (p. e. Texas Assessment of Knowledge and Skills - TAKs-). Se plantea que prevalecen visiones hegemónicas de las prácticas letradas, ajenas a los estudiantes, por ello, se retoman los NEL y los conceptos de comunidad de práctica y prácticas letradas híbridas para mostrar la alternancia de códigos y registros del hogar y la escuela, es decir, la combinación de prácticas vernáculas y oficiales. Se explica que dicha hibridez puede ser un recurso que promueve la literacidad (Gutiérrez et al., citado por De la Piedra).
María del Carmen Lorenzatti, por su parte, presenta los resultados de una investigación que indagó por las prácticas letradas que desarrollan adultos de nula/ baja escolaridad en distintos espacios sociales (religioso, doméstico, financiero, laboral y escolar). Se retoman las categorías de literacidad, actividad, multimodalidad y apropiación de conocimiento para mostrar cómo los adultos son capaces de movilizar sus conocimientos y saberes para resolver asuntos de su vida cotidiana. El estudio evidencia cómo se usa la tecnología en función de situaciones sociales y cómo los adultos otorgan sentido a los textos, de acuerdo a sus actividades, a usos específicos, a destrezas y a su conocimiento social.

Los estudios incluidos en este libro se realizan desde un enfoque sociocultural, los cuales evidencian el problema de la desigualdad social y cultural, manifiesta en la distribución social y el acceso a las prácticas de literacidad, que ha sido uno de los principales propósitos de la escuela moderna. Se muestra que la lectura y la escritura se constituyen en una fuente para comprender la complejidad de las realidades culturales, además de su relación con el desempeño escolar. También resulta fundamental entender que el acceso al dominio de la cultura escrita como lo señala Petrucci (2003, p. 27) “instaura una relación tajante entre aquel que escribe y aquel que no, entre aquel que lee y aquel que no, entre el que lo hace bien y mucho y el que lo hace mal y poco", en ese sentido, el abordaje histórico de la desigualdad a la que se alude, se asocia a las estrategias de distribución del poder político, económico, sociocultural y las apuestas de los sistemas educativos, a fin de contrarrestar el fracaso escolar.

Los estudios incluidos en este libro, desde perspectivas epistemológicas alineadas con los NEL, son un aporte interesante para las prácticas de enseñanza y aprendizaje del lenguaje. Especialmente se busca profundizar en las formas de aprendizaje de individuos, grupos de individuos y prácticas colectivas de comunidades específicas. En ese sentido, el concepto de aprendizaje se liga al de práctica social y cultural. Además, permiten comprender que los procesos de enseñanza y aprendizaje no sólo se dan 
en la interacción en el aula, sino también fuera de ella, es decir, que la investigación sobre prácticas letradas aborda realidades in situ, esto es, el aprendizaje en un contexto natural situado (Cole, 1996). $\mathrm{Y}$ en esa perspectiva, los estudios permiten conocer estas prácticas desde dentro y procuran aportar a su comprensión y transformación. Cobran sentido, entonces, el rol de los estudiantes (sus intereses, el acceso a materiales bibliográficos, sus posibilidades como lectores y escritores), el rol del maestro (su experiencia como lector y escritor, su concepciones sobre el lenguaje, su apuesta didáctica), los textos (tipologías textuales, géneros, formatos) y cómo estos se integran y se articulan en la formulación de marcos conceptuales y metodológicos en la enseñanza.

Las poblaciones seleccionadas reflejan la diversidad particular del territorio americano, así como las diferentes maneras de aprender que los investigadores ponen en tensión con las formas estandarizadas ideológicamente, sobre todo, con respecto a las formas de conocimiento de las poblaciones indígenas que todavía perviven. Estos trabajos visibilizan a las poblaciones indígenas a la luz de los NEL, para recuperarlas y analizarlas de cara a las prácticas letradas hegemónicas. La particularización sobre las formas de aprendizaje y la observación sobre la diversidad de formas de lectura y escritura que se desarrollan en diferentes lugares de América son uno de los principales aportes de esta compilación de investigaciones muy bien articuladas y estructuradas. Se ofrece una mirada crítica de la relación entre cultura, procesos educativos, prácticas letradas y aprendizaje en la que se asume la diversidad en las formas y estilos de aprender como un potencial, así como la necesidad de atender a esta en el trabajo pedagógico como parte fundamental de las agendas educativas de países latinoamericanos en los que ha prevalecido el enfoque deficitario de enseñanza de las prácticas de literacidad.

\section{Referencias}

Cole, M. (1996). Cultural psychology. A once and future discipline. Cambridge, ma: Harvard University Press.

Frisancho, S., Moreno, M.T., Ruiz, P. y Zavala, V. (Eds.). (2017 [2011]). Aprendizaje, cultura y desarrollo. Una aproximación interdisciplinaria. Lima: Fondo Editorial Pontificia Universidad Católica del Perú.

Gumperz, J. \& D. Hymes (Eds) (1972). Directions in Sociolinguistics: The Ethnography of Communication. Oxford: Blackwell.

Petrucci, A. (2003). La ciencia de la escritura. Primera lección de paleografía. México: Fondo de Cultura Económica.

Street, B. (2005). Navigating Numeracies: Home/School Numeracy Practices. Multiple Perspectives on Attainment in Numeracy. Dordrecht, Netherlands: Springer.

Zavala, V., Niño-Murcia M. y Ames, P. (Eds.) (2004). Escritura y sociedad. Nuevas perspectivas teóricas y etnográficas. Lima: Red para el desarrollo de las ciencias sociales en el Perú.

How to reference this article: Yepes-Serrano, R. (2019). Aprendizaje, cultura y desarrollo. Una aproximación interdisciplinaria: reseña. Íkala, Revista de Lenguaje y Cultura, 24(2), 445-449. doi: 10.17533/ udea.ikala.v24n02a14 